论 文 有机半导体与有机发光专刊

\title{
二苯磷酰基取代四苯基硅的合成及在构筑宽禁带 聚合物中的应用
}

\author{
王储, 刘贺, 胡德华, 路萍*, 马於光 \\ 吉林大学超分子结构与材料国家重点实验室，长春 130012 \\ *通讯作者, E-mail: lup@jlu.edu.cn
}

收稿日期: 2013-01-05; 接受日期: 2013-01-28; 网络版发表日期: 2013-02-23 doi: 10.1360/032013-43

\begin{abstract}
摘要设计并合成了二苯磷酰基取代的四苯基硅基团, 并将其作为宽禁带聚合物母体材料 构筑基元，通过 Suzuki 反应偶连 3, 6 位取代的味唑合成了聚合物 SiCzP. 对聚合物的结构进 行了系统的表征. 与模型聚合物 $\mathrm{SiCz}$ 相比, 二苯磷酰基的强吸电子能力, 降低聚合物母体材 料的 LUMO 能级, 更有利于电子的注入. $\mathrm{SiCzP}$ 与 $\mathrm{SiCz}$ 的玻璃化转变温度分别为 219 与 $227{ }^{\circ} \mathrm{C}$, 失重 $5 \%$ 时的分解温度分别为 441 与 $426{ }^{\circ} \mathrm{C}$. 二者均具有良好的成膜性, 掺杂器件初 步结果表明, 二苯磷酰基的引入使器件的亮度和效率都得到提高, 其最大流明效率和功率效 率比 $\mathrm{SiCz}$ 分别提高了 $98 \%$ 和 $75 \%$.
\end{abstract}

关键词

宽禁带

聚合物

母体材料

Suzuki 反应

\section{1 引言}

有机宽禁带共轭聚合物是一类具有较宽 HOMOLUMO 能隙 $(>3.0 \mathrm{eV})$ 的聚合物, 通常颜色较浅, 光 激发时发射紫外或蓝紫的苂光. 它可广泛应用于电 致发光、紫外光探测和高密度存储等领域. 其科学意 义更在于宽禁带材料的发展. 采用掺杂技术, 利用分 子间的能量转移, 可以发展其他颜色的发光材料与器 件. 因此宽禁带聚合物的高能量发光具有重要价值.

宽禁带共轭聚合物结构设计的关键是控制有效 共轭长度. 目前高分子电致发光材料结构大部分是 通过不同 $\pi$ 共轭单体间共聚得到的 ${ }^{[1]}$. 宽禁带共轭聚 合物通常可采用三种方法构筑: 一是将共轭发光单 元通过非共轭的柔性链连接, 即在主链上嵌入柔性间 隔基团, 使得聚合物的能隙得以增加 ${ }^{[2]}$; 二是引入具 有扭曲结构的共轭单元 ${ }^{[3]}$, 降低其有效共轭长度, 调 控发光波长. 但这两种方式都会在一定程度上降低主
链上的载流子迁移率, 进而降低器件效率; 三是引入 其他非 $\pi-\pi$ *共轭单元, 如引入硅原子增宽带隙 ${ }^{[4]}$.

四苯基硅衍生物具有特殊的四面体结构, 可以有 效地打断共轭，增加带隙宽度，并且可以抑制分子间 的相互作用使含硅化合物具有较好的溶解性、热力学 性能和成膜性. 处于第三周期的硅原子较第二周期的 碳原子最外层多了 $\mathrm{d}$ 轨道，与 $\pi$ 共轭体系形成 $\sigma-\pi *$ 共轭 的同时, 最外层的 $\mathrm{d}$ 轨道与 $\pi$ 共轭体系也存在部分重 叠, 使聚合物的 LUMO 能级降低, 有利于电子的注入 ${ }^{[5]}$. 此外, 硅原子半径较大, 形成的键容易极化, 键能低, 相比较以碳为中心的化合物, 合成时较容易引入其他 的特定官能团来调节化合物的性质. 这些都使四苯基 硅衍生物成为理想的宽禁带材料的构筑基元 ${ }^{[6-9]}$.

有机宽禁带聚合物的重要应用之一是作为高效 率磷光染料的材料. 磷光材料在发光过程中可以利 用单线态和三线态激子, 理论上内量子效率可达到 $100 \%{ }^{[10]} .1998$ 年, 吉林大学率先采用了锇配合物 
$\left(\mathrm{Os}(\mathrm{CN})_{2}\left(\mathrm{PPh}_{3}\right)_{2} \mathrm{bpy}\right)$ 掺杂到聚乙烯基咔唑(PVK)中制 备了首个电致磷光器件, 并提出其内量子效率突破 $25 \%$ 的可能 ${ }^{[11,12]}$, 自此之后高效的母体与磷光材料 都已取得了长足的进步 ${ }^{[13]}$. 然而, 磷光材料在聚集态 发光淬灭, 所以通常将其以低浓度的形式掺杂到母 体材料中制作电致发光器件. 母体材料是整个发光 层的承载主体, $90 \%$ 的载流子都是在母体中传输的, 因而母体材料的注入与传输性质将直接影响到整个 器件的效果. 母体材料通常有三点要求: (1) 要有高 于客体的三线态能级, 防止能量从客体转移回母体; （2）要有良好的载流子传输性能; (3) 要有良好的热 学及形貌学稳定性.

在红、绿、蓝三基色磷光主体材料中, 最难解决 的就是蓝光的主体材料 ${ }^{[14,15]}$. 蓝色磷光染料的本体 发光在 $440 \mathrm{~nm}$ 左右, 要求主体的能隙更宽, 三线态 能级要达到 $2.7 \mathrm{eV}$ 以上. 尽管文献中报道一些特殊 载流子传输官能团的引入改善了材料的载流子传输 性能, 但是分子内电荷转移的出现, 使很多材料的三 线态能量不能达到所需的高度而不能用于蓝色磷光 掺杂或者效率受到影响 ${ }^{[16]}$, 因此, 目前可作为蓝色磷 光主体的聚合物依然缺乏. 我们选取 3,6 位取代的咔 唑作为聚合物骨架结构, 偶合二苯磷酰基取代的四 苯基硅单体, 设计并合成了 $\mathrm{SiCzP}$ 聚合物双极性母体 材料. 咔唑 9 位上连接含六个碳的烷基链以进一步增 强聚合物的溶解性和成膜性. 同时主链上的咔唑基团, 可以改善聚合物的空穴注入性能. 本文介绍了 $\mathrm{SiCzP}$ 聚合物的合成、表征与器件性质的初步研究, 并与不 含二苯磷酰基团的聚合物 $\mathrm{SiCz}$ 进行了系统的比较.

\section{2 聚合物的合成与结构表征}

聚合物单体的合成路线如图 1 所示, 通过对反应 条件的选择与摸索, 我们得到了最优反应条件, 各步 产物的产率均比较理想. M1 用之前报道的常规方法 合成 ${ }^{[11]}$. M5 是一个新型含硅化合物, 通过对溴苯与 三氯苯硅反应得到三澳代四苯基硅单体 M4. 之后丁 基锂引发, M4 与二苯基氯化磷反应, 过氧化氢氧化 后得到单体 M5. 聚合物的合成均由其相应的单体采 用经典的 Suzuki 偶联反应得到. 对于 Suzuki 偶联反 应, 反应的温度、时间、催化剂的种类、催化剂的量、 单体浓度对聚合物的聚合程度, 即产物分子量影响 很大. 为了得到较高分子量的聚合物产物, 我们通过
控制变量法, 调控催化剂的摩尔比例, 改变反应单体 的浓度、反应温度等条件, 最终得到了两种聚合物. $\mathrm{SiCz}$ 的重均分子量为 16000 , 分散度为 $1.51 ; \mathrm{SiCzP}$ 的 重均分子量为 11000 , 分散度为 2.19. 两个聚合物均 可溶于常规的有机溶剂, 如四氢呋喃、甲苯、氯仿等.

\section{$2.13,6$-(4,4,5,5-四甲基-1,3,2-二氧杂硼)-9- 己基- 9H-咔唑(M2)}

将 $2.5 \mathrm{~g}(6.2 \mathrm{mmol}) \mathbf{M 1}$ 溶于 $100 \mathrm{~mL}$ 新蒸无水四 氢呋喃中, 经脱气处理三次后, 置于低温反应仪中, $-78{ }^{\circ} \mathrm{C}$ 搅拌 $10 \mathrm{~min}$ 后逐滴加入 $5.2 \mathrm{~mL}(2.4 \mathrm{~mol} / \mathrm{L}$, $12.4 \mathrm{mmol}$ ) 正丁基锂. $-78{ }^{\circ} \mathrm{C}$ 下活化 $2 \mathrm{~h}$ 后逐滴加入 $3.2 \mathrm{~mL}$ (15.5 mmol) 2-异丙氧基-4,4,5,5-四甲基-1,3,2-二 氧杂硓烷, 再升至室温继续反应 48 h. 之后将溶液倒入 水中淬灭反应, 用无水乙醚萃取三次, 收集的有机相用 无水硫酸镁干燥过夜. 过滤, 浓缩溶液, 以二氯甲烷石油醚混合溶剂为洗脱剂, 薄层层析得到白色固体 $1.58 \mathrm{~g}$, 产率 $56 \% .{ }^{1} \mathrm{H} \mathrm{NMR}\left(\mathrm{CDCl}_{3}, 500 \mathrm{MHz}\right): \delta(\mathrm{ppm})$ $8.66(\mathrm{~s}, 2 \mathrm{H}, \mathrm{Ar}-\mathrm{H}), 7.91 \sim 7.89(\mathrm{~d}, 2 \mathrm{H}, J=8.2 \mathrm{~Hz}$, $\mathrm{Ar}-\mathrm{H}), 7.39 \sim 7.38$ (d, $2 \mathrm{H}, J=7.9 \mathrm{~Hz}, \mathrm{Ar}-\mathrm{H}), 4.32 \sim 4.29$ (t, $\left.2 \mathrm{H}, J=6.7 \mathrm{~Hz}, \mathrm{CH}_{2}\right), 1.86 \sim 1.83\left(\mathrm{~m}, 2 \mathrm{H}, \mathrm{CH}_{2}\right), 1.39$ (s, $\left.24 \mathrm{H}, \mathrm{CH}_{3}\right), 1.35 \sim 1.32\left(\mathrm{~m}, 2 \mathrm{H}, \mathrm{CH}_{2}\right), 1.30 \sim 1.24(\mathrm{~m}, 4 \mathrm{H}$, $\left.\mathrm{CH}_{2}\right), 0.86 \sim 0.83$ (t, $\left.3 \mathrm{H}, J=7.0 \mathrm{~Hz}, \mathrm{CH}_{3}\right)$. FTIR $\left(\mathrm{cm}^{-1}\right)$ : 2961 (s), 1868 (s), 1597 (m), 1469 (m), 1356 (s), $1252(\mathrm{~m}), 1164(\mathrm{~s}), 1076(\mathrm{~m}), 852(\mathrm{~m}), 812(\mathrm{~m}), 752(\mathrm{w})$, 662 (s). 元素分析: 理论值 $\mathrm{C}_{30} \mathrm{H}_{43} \mathrm{~B}_{2} \mathrm{NO}_{4}: \mathrm{C}, 71.59 ; \mathrm{H}$, $8.61 ; \mathrm{N}, 2.78$. 实测值: $\mathrm{C}, 71.76 ; \mathrm{H}, 8.40 ; \mathrm{N}, 2.70$.

\section{2 双-(4-溴苯基)-二苯基硅(M3)}

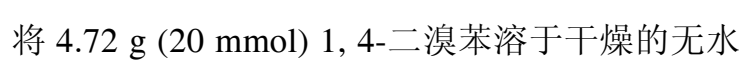
乙醚中, 经脱气处理三次后, $0{ }^{\circ} \mathrm{C}$ 下摚拌 $10 \mathrm{~min}$ 后用 注射器逐滴加入 $8.4 \mathrm{~mL}(2.5 \mathrm{~mol} / \mathrm{L}, 21 \mathrm{mmol})$ 正丁基 锂, 体系在 $0{ }^{\circ} \mathrm{C}$ 下活化 $2 \mathrm{~h}$ 后, 再逐滴加入 $2.1 \mathrm{~mL}$ (10 mmol)二氯二苯基硅, 后升至室温搅拌 $12 \mathrm{~h}$. 将 溶液倒入水中淬灭反应, 用无水乙醚萃取三次, 收集 到的有机相用无水硫酸镁干燥过夜. 过滤, 浓缩溶液, 粗产物用无水乙醇和二氯甲烷重结晶得到白色固体 $3.46 \mathrm{~g}$, 产率 $70 \% .{ }^{1} \mathrm{H} \mathrm{NMR}\left(\mathrm{CDCl}_{3}, 500 \mathrm{MHz}\right): \delta(\mathrm{ppm})$ $7.46 \sim 7.44(8 \mathrm{H}, \mathrm{m}, \mathrm{Ar}-\mathrm{H}), 7.40 \sim 7.37(2 \mathrm{H}, \mathrm{t}, J=7.6 \mathrm{~Hz}$, $\mathrm{Ar}-\mathrm{H}), 7.33 \sim 7.30(8 \mathrm{H}, \mathrm{m}, \mathrm{Ar}-\mathrm{H}) .{ }^{13} \mathrm{C} \mathrm{NMR}\left(\mathrm{CDCl}_{3}\right.$, $125 \mathrm{MHz}): \delta(\mathrm{ppm}) 140.38,138.77,135.56,135.21$, 133.78, 132.56, 130.66, 127.53. FTIR $\left(\mathrm{cm}^{-1}\right): 3025(\mathrm{~s})$, 1969 (w), 1915 (m), 1807 (w), 1565 (s), 1473 (s), 1424 

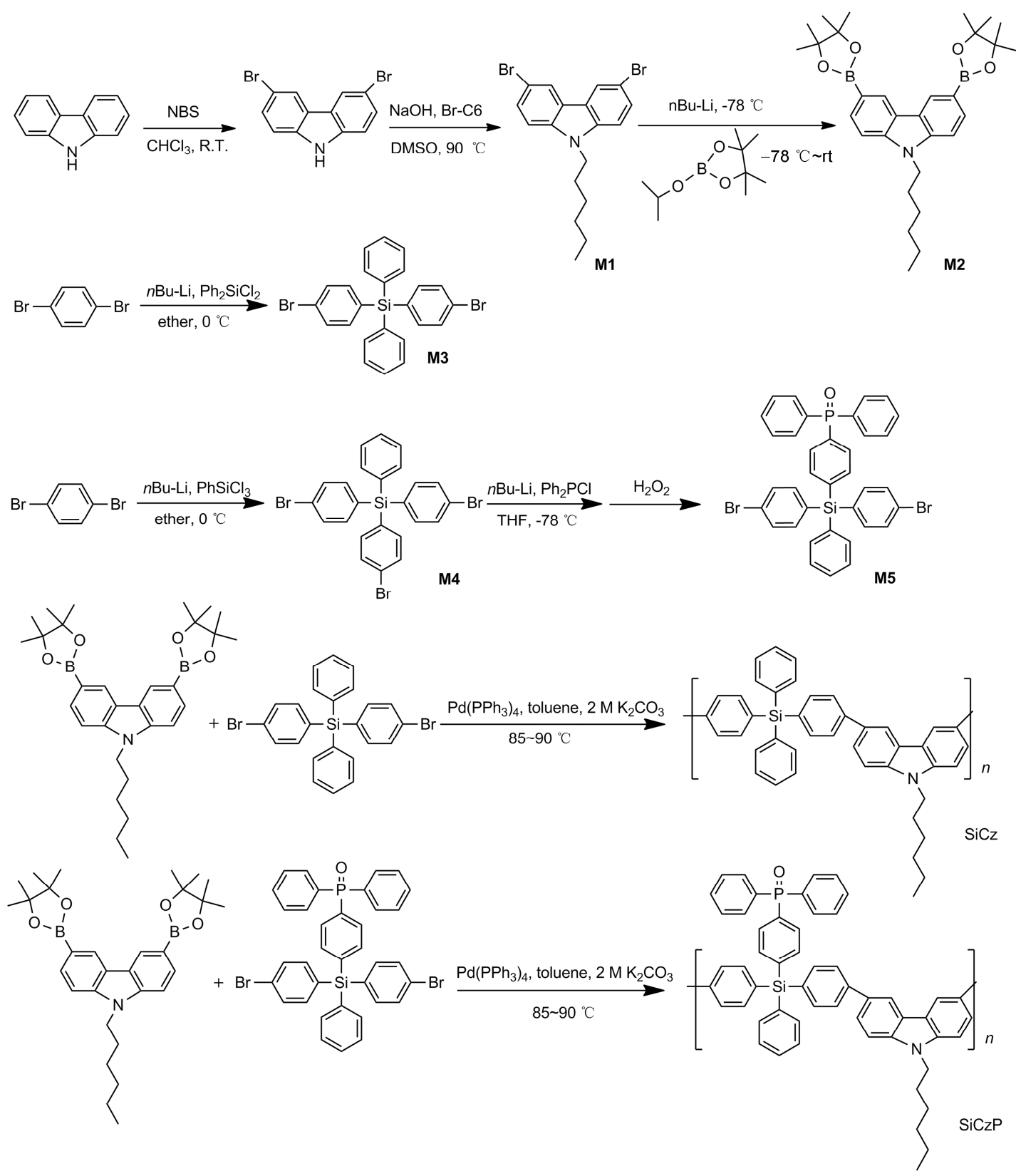

图 1 单体与聚合物的合成路线

(s), 1378 (s), 1062 (s), 1106 (s), 1010 (s), 807 (s), 713

(s), 521 (s). 元素分析: 理论值 $\mathrm{C}_{24} \mathrm{H}_{18} \mathrm{Br}_{2} \mathrm{Si}: \mathrm{C}, 58.32$; H, 3.67. 实测值: C, 58.66; H, 3.66.

\section{3 三-(4-溴苯基)-苯基硅(M4)}

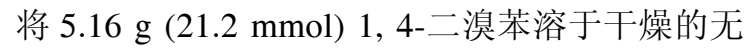
水乙醚, 经脱气处理三次后, $0{ }^{\circ} \mathrm{C}$ 下搅拌 $10 \mathrm{~min}$ 后用 注射器逐滴加入 $9.9 \mathrm{~mL}(2.4 \mathrm{~mol} / \mathrm{L}, 24 \mathrm{mmol})$ 正丁基
锂, 体系在 $0{ }^{\circ} \mathrm{C}$ 下活化 $2 \mathrm{~h}$ 后, 再逐滴加入 $1.13 \mathrm{~mL}$ 氯化一苯基硅, $0{ }^{\circ} \mathrm{C}$ 搅拌 $12 \mathrm{~h}$. 将溶液倒入水中淬灭 反应，用无水乙醚萃取三次，收集到的有机相用无水 硫酸镁干燥过夜. 过滤, 浓缩溶液, 粗产物用无水乙 醇和二氯甲烷重结晶得到白色固体 $3.02 \mathrm{~g}$, 产率 $63 \%$.

${ }^{1} \mathrm{H}$ NMR (500 MHz, $\mathrm{CDCl}_{3}$ ): $\delta(\mathrm{ppm}) 7.534 \sim 7.517(6 \mathrm{H}$, $\mathrm{d}, J=8.197, \mathrm{Ar}-\mathrm{H}), 7.491 \sim 7.449(3 \mathrm{H}, \mathrm{m}, \mathrm{Ar}-\mathrm{H})$, 7.406 7.391 (2H, d, J= 7.409, Ar-H), 7.370 7.354 (6H, 
d, $J=7.724$, Ar-H). FTIR $\left(\mathrm{cm}^{-1}\right): 3034$ (w), 1909 (w), $1800(\mathrm{w}), 1644$ (w), 1570 (s), 1429 (m), 1373 (s), 1305 (w), $1185(\mathrm{~m}), 1117$ (s), 1068 (s), 1008 (s), 812 (s), 724 (s), $534(\mathrm{~s})$.

\section{4 双溴-(4-二苯基磷酰苯基)-二苯基硅(M5)}

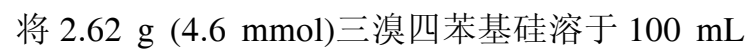
新蒸无水四氢呋喃中, 经脱气处理三次后, 转移至低 温反应仪中, $-78{ }^{\circ} \mathrm{C}$ 下搅拌 $10 \mathrm{~min}$ 后用注射器逐滴加 入 $1.9 \mathrm{~mL}(2.4 \mathrm{~mol} / \mathrm{L}, 4.6 \mathrm{mmol})$ 正丁基锂, 体系在 $-78{ }^{\circ} \mathrm{C}$ 下活化 $2 \mathrm{~h}$ 后, 再逐滴加入 $1 \mathrm{~mL}(5.1 \mathrm{mmol})$ 一 氯二苯基磷, 后升至室温搅拌 $48 \mathrm{~h}$. 将溶液倒入水中 淬灭反应, 用二氯甲烷萃取三次, 收集到的有机相经 旋转蒸发装置去除大部分多余溶剂, 将剩余少量液 体溶于二氯甲烷中, 慢慢加入约 $30 \mathrm{~mL}$ 的 $30 \%$ 双氧水, 室温下搅拌 $3 \mathrm{~h}$. 将溶液倒入水中淬灭反应, 用二氯 甲烷萃取三次, 收集到的有机相用无水硫酸镁干燥 过夜. 过滤, 浓缩溶液, 粗产物用无水石油醚-乙酸乙 酯混合溶剂为洗脱剂, 柱层析得到白色固体 $1.64 \mathrm{~g}$, 产率 53\%. ${ }^{1} \mathrm{H}$ NMR $\left(\mathrm{CDCl}_{3}, 500 \mathrm{MHz}\right): \delta(\mathrm{ppm})$ 7.709 7.605 (8H, m, Ar-H), 7.570 7.440 (13H, m, $\mathrm{Ar}-\mathrm{H}), 7.409 \sim 7.360(6 \mathrm{H}, \mathrm{m}, \mathrm{Ar}-\mathrm{H}) .{ }^{13} \mathrm{C} \mathrm{NMR}\left(\mathrm{CDCl}_{3}\right.$, $125 \mathrm{MHz}): \delta$ (ppm) 138.31, 137.87, 136.19, 134.87, 134.05, 132.57, 132.15, 132.07, 131.76, 131.41, 130.32, 128.62, 128.52, 128.28, 125.32. FTIR $\left(\mathrm{cm}^{-1}\right): 3358(\mathrm{~m})$, 3053 (m), 2966 (m), 1901 (w), 1682 (w), 1571 (s), 1482 (m), 1437 (m), 1373 (m), 1191 (s), 1130 (s), 1062 (s), $1010(\mathrm{~s}), 812(\mathrm{~s}), 729(\mathrm{~s}), 568(\mathrm{~s})$. 元素分析: 理论值 $\mathrm{C}_{36} \mathrm{H}_{27} \mathrm{Br}_{2} \mathrm{OPSi}$ : $\mathrm{C}, 62.26$; H, 3.92. 实测值: C, 62.44; $\mathrm{H}, 4.12$.

\subsection{SiCz 的合成}

将 $160 \mathrm{mg}(0.32 \mathrm{mmol}) \mathbf{M 2}, 158 \mathrm{mg}(0.32 \mathrm{mmol})$ $\mathbf{M 3}$ 和 $5.5 \mathrm{mg}\left(\mathrm{PPh}_{3}\right)_{4} \mathrm{Pd}(0)$ 催化剂溶于 $2.2 \mathrm{~mL}$ 甲苯和 $1.4 \mathrm{~mL}$ 浓度为 $2 \mathrm{~mol} / \mathrm{L}$ 的碳酸钾溶液中, 体系经脱气 处理三次后转入油浴, $90{ }^{\circ} \mathrm{C}$ 下反应 $72 \mathrm{~h}$. 将溶液倒 入水中淬灭反应, 用二氯甲烷萃取三次, 合并有机相 用无水硫酸镁干燥过夜. 过滤, 再将滤液快速过中性 $\mathrm{Al}_{2} \mathrm{O}_{3}$ 柱除去催化剂. 浓缩溶液, 在 $100 \mathrm{~mL}$ 无水甲醇 中沉淀, 过滤, 收集产物, 干燥, 得到灰白色固体 $140 \mathrm{mg}$, 产率 $63 \%$. 均重分子量为 16000 , 分散度为 1.51. ${ }^{1} \mathrm{H}$ NMR $\left(\mathrm{CDCl}_{3}, 600 \mathrm{MHz}\right): \delta(\mathrm{ppm}) 8.611 \sim 7.176$ $(24 \mathrm{H}, \mathrm{m}, \mathrm{Ar}-\mathrm{H}), 4.510 \sim 4.095(2 \mathrm{H}, \mathrm{m}, \mathrm{Ar}-\mathrm{H}), 2.098 \sim$
$0.639(11 \mathrm{H}, \mathrm{m}, \mathrm{Ar}-\mathrm{H}) .{ }^{13} \mathrm{C} \mathrm{NMR}\left(\mathrm{CDCl}_{3}, 150 \mathrm{MHz}\right)$ : $\delta(\mathrm{ppm}) 143.140,140.593,137.013,136.518,134.604$, 132.146, 131.938, 129.625, 127.957, 126.738, 125.416, 123.566, 119.037, 109.183, 43.392, 31.618, 29.067, 27.031, 22.588, 14.063. FTIR $\left(\mathrm{cm}^{-1}\right): 3058(\mathrm{~m}), 2923$ (s), $2855(\mathrm{~m}), 1587(\mathrm{~s}), 1473(\mathrm{~s}), 1426(\mathrm{~m}), 1350(\mathrm{~m})$, $1283(\mathrm{~m}), 1189(\mathrm{~m}), 1114(\mathrm{~s}), 803(\mathrm{~s}), 702(\mathrm{~s}), 639(\mathrm{~m})$, 545 (s). 元素分析：理论值 $\mathrm{C}_{42} \mathrm{H}_{39} \mathrm{NSi}: \mathrm{C}, 86.11 ; \mathrm{H}$, 6.71; N, 2.39. 实测值: C, 83.03; H, 6.43; N, 2.33 .

\section{6 $\mathrm{SiCzP}$ 的合成}

将 $160 \mathrm{mg}(0.32 \mathrm{mmol}) \mathbf{M} 2,222 \mathrm{mg}(0.32 \mathrm{mmol})$ M5 和 $7.6 \mathrm{mg}\left(\mathrm{PPh}_{3}\right)_{4} \mathrm{Pd}(0)$ 催化剂溶于 $3.2 \mathrm{~mL}$ 甲苯和 $2.2 \mathrm{~mL}$ 浓度为 $2 \mathrm{~mol} / \mathrm{L}$ 的碳酸钾溶液中, 体系经脱气 处理三次后转入油浴, $90{ }^{\circ} \mathrm{C}$ 下反应 $72 \mathrm{~h}$. 将溶液倒 入水中淬灭反应, 用二氯甲烷萃取三次, 合并有机相 用无水硫酸镁干燥过夜. 过滤, 再将滤液快速过中性 $\mathrm{Al}_{2} \mathrm{O}_{3}$ 柱除去催化剂. 浓缩溶液, 在 $100 \mathrm{~mL}$ 无水甲醇 中沉淀, 过滤, 收集产物, 干燥, 得到灰白色固体 $135 \mathrm{mg}$, 产率 $60 \%$. 均重分子量为 11000 , 分散度为 2.19. ${ }^{1} \mathrm{H}$ NMR $\left(\mathrm{CDCl}_{3}, 600 \mathrm{MHz}\right) ： \delta$ (ppm) 8.709 $7.069(33 \mathrm{H}, \mathrm{m}, \mathrm{Ar}-\mathrm{H}), 4.575 \sim 3.866(2 \mathrm{H}, \mathrm{m}, \mathrm{Ar}-\mathrm{H})$, 2.105 0.687 $(11 \mathrm{H}, \mathrm{m}, \mathrm{Ar}-\mathrm{H}) .{ }^{13} \mathrm{C}$ NMR $\left(\mathrm{CDCl}_{3}, 150\right.$ MHz): $\delta(\mathrm{ppm}) 143.435,140.629,136.956,136.421$, $136.320,132.190,132.119,131.967,131.250,131.182$, $129.903,128.572,128.479,128.087,126.855,125.428$, $123.539,119.022,109.213,43.406,31.597,29.053$, 27.012, 22.566, 14.037. FTIR $\left(\mathrm{cm}^{-1}\right): 3059(\mathrm{~m}), 2929$ (s), $2855(\mathrm{~m}), 1595(\mathrm{~s}), 1473(\mathrm{~s}), 13371(\mathrm{w}), 1283(\mathrm{w})$, $1194(\mathrm{~m}), 1114$ (s), 1067 (m), 803 (s), 694 (s), 647 (m), 566 (s). 元素分析：理论值 $\mathrm{C}_{54} \mathrm{H}_{48}$ NOPSi: C, 82.51; $\mathrm{H}, 6.16 ; \mathrm{N}, 1.78$. 实测值: $\mathrm{C}, 80.34 ; \mathrm{H}, 6.21 ; \mathrm{N}, 2.12$.

\section{3 结果与讨论}

\section{1 聚合物的光物理性质}

聚合物在四氢呋喃溶液中 $\left(1 \times 10^{-5} \mathrm{~mol} / \mathrm{L}\right)$ 的吸收 和发射光谱如图 2 所示. 可以看到, 这两个聚合物的 吸收和发射峰非常相似. 它们的最大吸收峰位于 302 $\mathrm{nm}$ 处, 归属于主链上苯基与咔唑所形成的 $\pi$ 共轭的 $\pi-\pi *$ 跃迁, 最大发射峰位于 $393 \mathrm{~nm}$ 处, 在 375 和 420 $\mathrm{nm}$ 处都有一个肩峰. 聚合物溶液的吸收与发射主峰 均处于紫外区域, 说明四苯基硅有效地打断主链的 


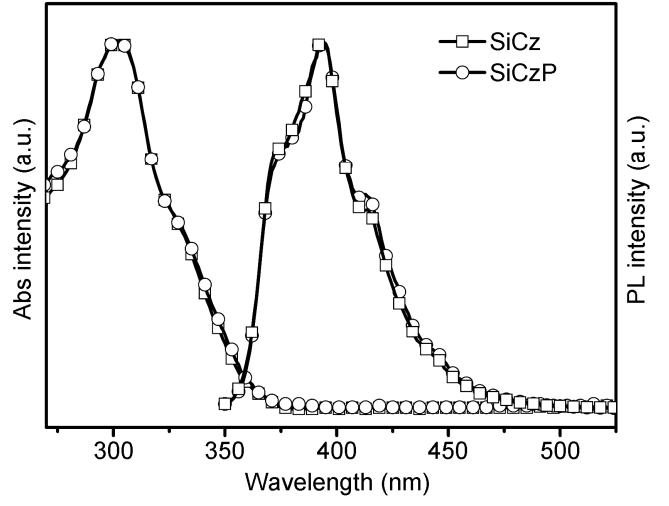

图 $2 \mathrm{SiCz}$ 与 $\mathrm{SiCzP}$ 在 THF 中的紫外吸收与荧光发射光谱 (激发波长 $323 \mathrm{~nm}$, 聚合物浓度 $1 \times 10^{-5} \mathrm{~mol} / \mathrm{L}$ )

有效共轭长度, 使聚合物具有较高的禁带宽度; 其次, 侧链上以非共轭的方式引入二苯基磷酰基并没有影 响聚合物的禁带宽度. 聚合物的发射光谱没有出现 红移, 这样不会改变聚合物的三线态能级. 从聚合物 的吸收带边计算其禁带宽度为 $3.42 \mathrm{eV}$.

\section{2 聚合物的热力学性质}

对于光电材料来说, 由于其应用于器件的过程 中, 会有焦耳热产生, 如果材料本身的热性质较差, 将直接影响器件的寿命. 图 3 给出了这两个聚合物 的差示扫描量热分析(DSC)曲线(第二次加热循环). 从图中我们可以观察到 $\mathrm{SiCz}$ 与 $\mathrm{SiCzP}$ 均具有较高的 玻璃化转变温度, 分别为 227 和 $219{ }^{\circ} \mathrm{C}$. 图 4 所示 为聚合物的热重曲线. 从图中我们可以看到 $\mathrm{SiCz}$ 与 $\mathrm{SiCzP}$ 均具有较高的热分解温度, 失重 $5 \%$ 时的温度 分别为 426 和 $441{ }^{\circ} \mathrm{C}$. 两个化合物良好的热稳定性 对于薄膜的稳定性以及电致发光器件的稳定性都是 很重要的.

\section{3 聚合物的电化学性质}

使用循环伏安方法来研究化合物的电化学性质, 通过计算可以得到它们的氧化还原电位及相应的能 级. 聚合物薄膜的循环伏安曲线如图 5 所示. 通过计 算得到了 $\mathrm{SiCz}$ 和 $\mathrm{SiCzP}$ 的 LUMO 能级分别为 -1.99 与 $-2.07 \mathrm{eV} . \mathrm{SiCzP}$ 的 LUMO 能级与 $\mathrm{SiCz}$ 的 LUMO 能级相比, 降低了 $0.08 \mathrm{eV}$, 说明二苯基磷酰基的引 入降低了母体材料的 LUMO 能级, 更有利于电子的 注入. 而 $\mathrm{SiCz}$ 和 $\mathrm{SiCzP}$ 的 $\mathrm{HOMO}$ 能级分别为 -5.61 与-5.62 eV, 表现为典型的咔唑的氧化特性, 二苯基

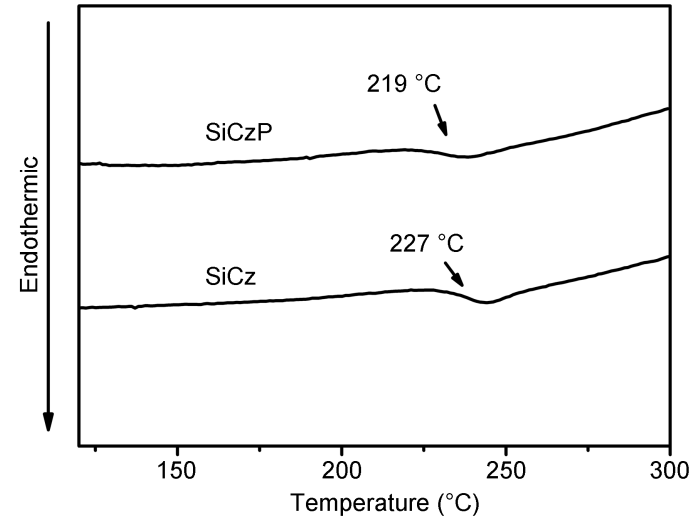

图 3 聚合物的 DSC 曲线

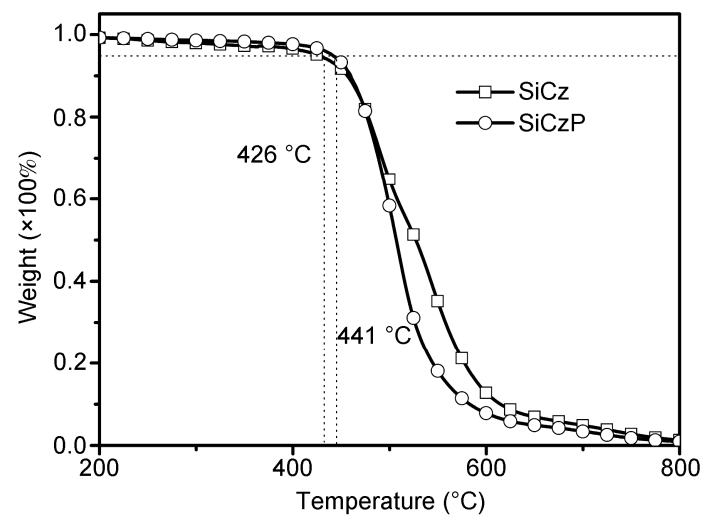

图 4 聚合物的热重曲线

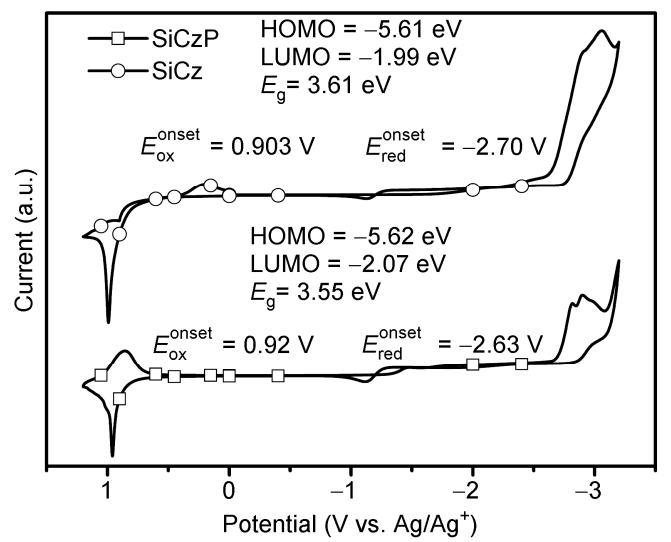

图 $5 \mathrm{SiCz}$ 与 $\mathrm{SiCzP}$ 的循环伏安曲线

磷酰基没有对 HOMO 能级产生影响.

\section{4 聚合物的薄膜形貌}

聚合物相对小分子一个非常重要的优势就是其 
可用溶液加工的方法制作器件, 这样可以简化器件 制备工艺，因而聚合物能否旋涂成高质量的薄膜是 其优势体现的关键. 将聚合物溶解在二氯甲烷溶剂 中, 浓度为 $10 \mathrm{mg} / \mathrm{mL}$, 以 $3000 \mathrm{rp} / \mathrm{min}$ 的转速旋涂在 石英基底上, 研究了其薄膜形貌. 如图 6 所示, 两种 聚合物均能形成平整连续的薄膜, $\mathrm{SiCz}$ 与 $\mathrm{SiCzP}$ 薄膜 的表面粗粘度 $(\mathrm{rms})$ 分别为 0.566 和 $0.523 \mathrm{~nm}$. 二苯 磷酰基的引入没有影响聚合物的成膜性. 对于磷光 主客体掺杂体系来说, 通常需要主体材料与磷光分 子之间具有良好的化学相容性. 因为一方面主-客体 之间分散性不好会抑制能量转移，同时聚集会导致 三线态间淬灭; 另一方面, 外主客体之间的相分离 会影响器件的寿命. 我们测量了其掺杂 $10 \mathrm{wt} \%$ 双 (4,6-二氟苯基吡啶- $N, C 2$ ) 吡啶甲酰合铱 (FIrpic) 的薄 膜形貌(图 6). 从图中可以观察到这两个聚合物与 FIrpic 之间具有很好的化学相容性, 薄膜平整连续, 并且没有出现相分离, 这对于其在器件中的应用是 非常必要的.

\section{5 聚合物的掺杂光谱}

在前面的光物理性质研究中, 我们发现这些聚 合均具有较宽的禁带, 但是磷光主客体的掺杂体系 除了满足光谱重叠之外, 还涉及主客体三线态能量 的问题. 我们直接将聚合物掺杂磷光配合物, 看是否
实现有效的能量转移. 图 7 所示为聚合物掺杂了 1 $\mathrm{wt} \%$ 和 $10 \mathrm{wt} \%$ 的 FIrpic 薄膜下的发射光谱, 从图中可 以看到, 在掺杂程度达到 $10 \%$ 时, $\mathrm{SiCzP}$ 与 FIrpic 之间 几乎实现完全的能量转移. 与 $\mathrm{SiCz}$ 相比 ${ }^{[17]}$, 表现出 的能量转移更完全.

\section{6 聚合物的电致发光性质初步研究}

在前面的掺杂光谱中, 我们研究了所得到的聚 合物是否能用于蓝色磷光配合物 FIrpic 的母体, 发现 $\mathrm{SiCzP}$ 能发生较为完全的能量转移, 因而我们将 $\mathrm{SiCz}$ 与 $\mathrm{SiCzP}$ 两种材料制作了结构为 ITO/PEDOT:PSS(60 $\mathrm{nm}) / \mathrm{Host}: \mathrm{FIrpic}(8 \mathrm{wt} \%, 55 \mathrm{~nm}) / \mathrm{TPBi}(50 \mathrm{~nm}) / \mathrm{LiF}(0.5$ $\mathrm{nm}) / \mathrm{Al}(200 \mathrm{~nm})$ 的电致发光器件, 初步研究了器件 的性质. 器件性能上所表现出来的规律与我们所设 计的思路是一致的, 即 $\mathrm{SiCzP}$ 由于载流子注入的优 势使其器件性能较 $\mathrm{SiCz}$ 有了一定程度的提高. 图 8 同时给出了这个器件的效率-电流密度曲线, 具体的 器件性能数据在表 1 中给出. 器件的开启电压比较 接近, 但从流明效率和外量子效率上看, $\mathrm{SiCzP}$ 作为 蓝色磷光材料 FIrpic 母体的性能要优于 $\mathrm{SiCz}$, 展示 了其在蓝光磷光器件中应用的潜力. 其最大流明效 率和功率效率分别为 $2.68 \mathrm{~cd} / \mathrm{A}$ 和 $0.51 \mathrm{~lm} / \mathrm{W}$, 相比 较于 $\mathrm{SiCz}$, 同样条件下的效率分别提高了 $98 \%$ 和 $75 \%$. 器件的发射光谱也较为稳定. 从器件的初步
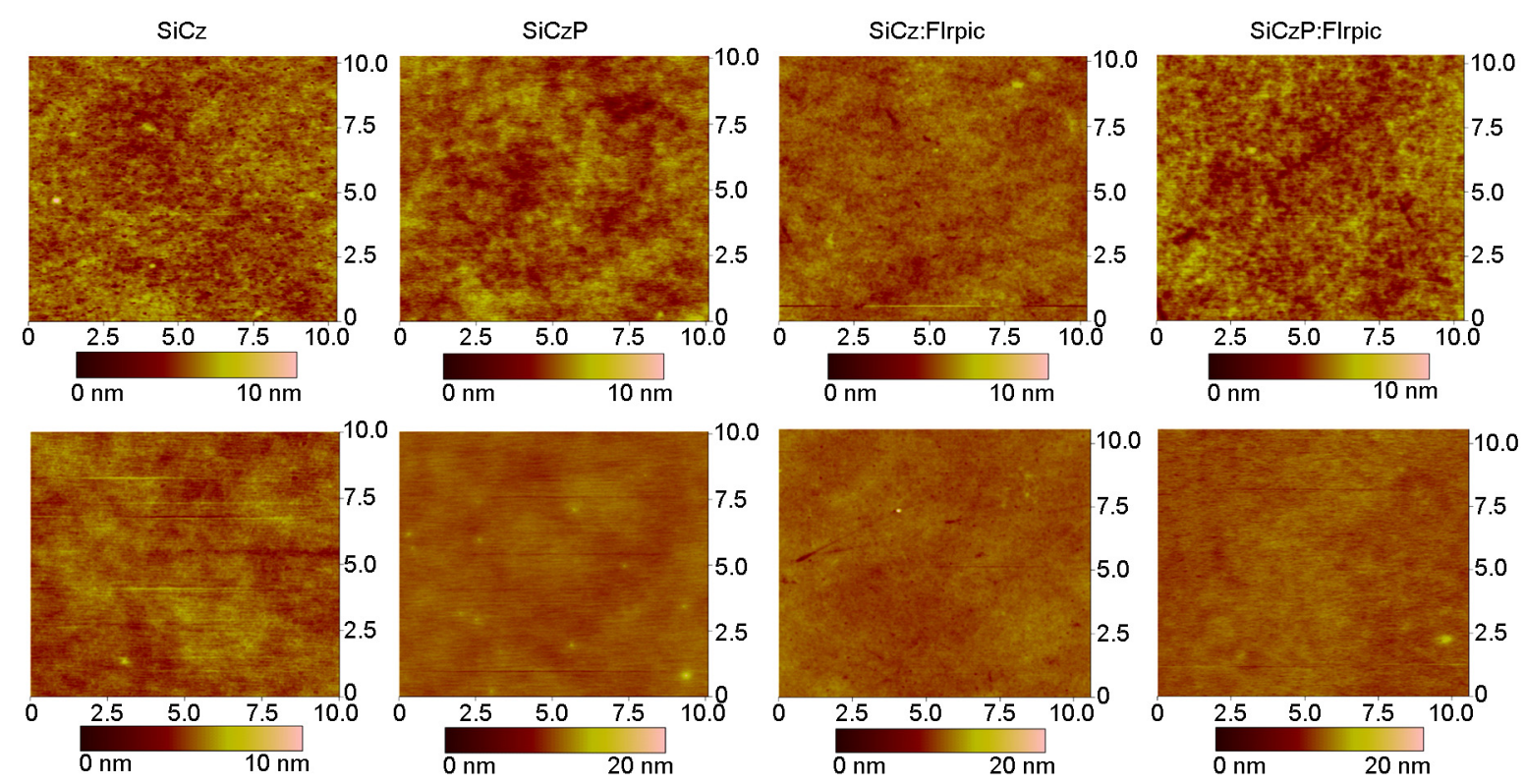

图 6 聚合物旋涂薄膜与掺杂薄膜热处理前(上排)后(下排)形貌 


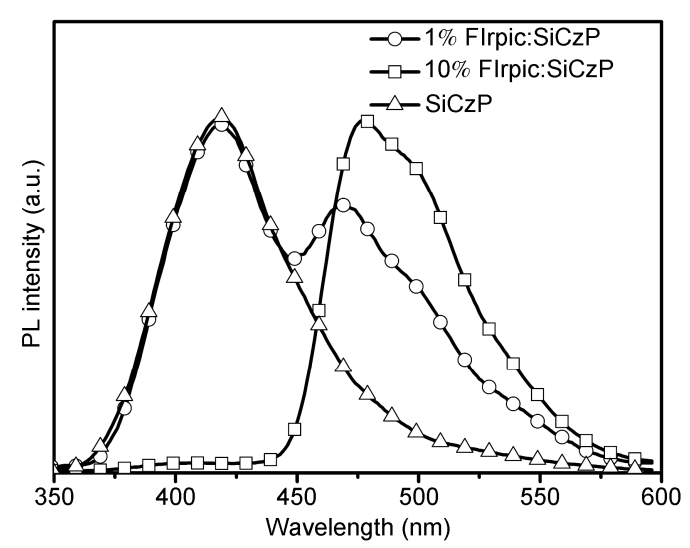

图 7 聚合物掺杂 FIrpic 的薄膜发射光谱

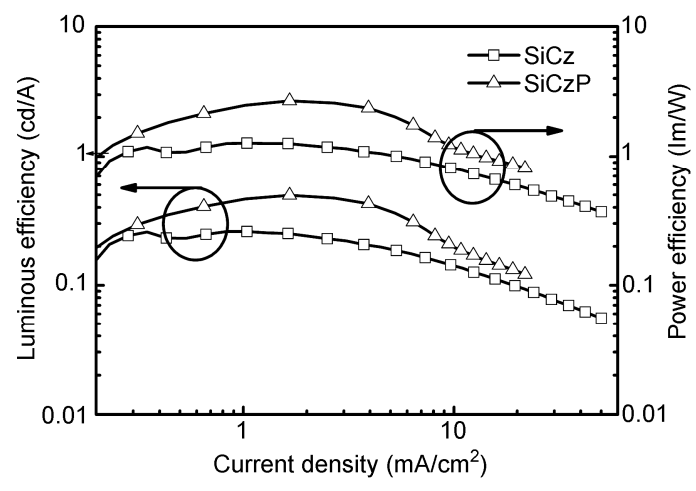

图 $8 \mathrm{SiCz}$ 与 $\mathrm{SiCzP}$ 的效率-电流密度曲线

\section{表 1 器件性能数据总结}

\begin{tabular}{ccccc}
\hline Host & $\begin{array}{c}\text { Turn-on } \\
\text { votage }(\mathrm{V})\end{array}$ & $\begin{array}{c}\text { Brightness } \\
\left(\mathrm{cd} / \mathrm{m}^{2}\right)\end{array}$ & $\begin{array}{c}\text { Luminous } \\
\text { efficiency } \\
(\mathrm{cd} / \mathrm{A})\end{array}$ & $\begin{array}{c}\text { Power } \\
\text { efficiency } \\
(\mathrm{lm} / \mathrm{W})\end{array}$ \\
\hline $\mathrm{SiCz}$ & 10 & 190 & 1.35 & 0.29 \\
$\mathrm{SiCzP}$ & 10 & 200 & 2.68 & 0.51 \\
\hline
\end{tabular}

结果发现, 聚合物载流子注入性质的改善确实可以 提高器件的效率.

\section{4 结论}

设计合成了新型二苯磷酰基取代四苯基硅基团, 并应用于宽禁带聚合物的构筑. 利用 Suzuki 偶连的方 法合成了具有相同禁带宽度、不同侧基的聚合物 $\mathrm{SiCz}$ 和 $\mathrm{SiCzP}$, 并通过核磁、红外、元素分析等方法对聚合 物进行了系统的表征. 两种聚合物均具有宽的禁带, 为蓝紫色发光材料, 且具有良好的热稳定性、成膜性 和掺杂行为. 电化学表征表明, 二苯磷酰基的引入会 降低聚合物的 LUMO 能级, 更有利于电子的注入.

初步研究了 $\mathrm{SiCz}$ 和 $\mathrm{SiCzP}$ 作为蓝色铱配合物母 体材料的器件性能, 母体材料禁带宽度的增大有利 于能量的转移, 二苯磷酰基的引入使器件的亮度和 效率都得到提高, $\mathrm{SiCzP}$ 的蓝色的掺杂器件均表现出 了比 $\mathrm{SiCz}$ 更好的性能.

\section{参考文献}

1 Ma YG, Zhang HY, Shen JC, Che CM. Electroluminescence from triplet metal-ligand charge-transfer excited state of transition metal complexes. Synth Met, 1998, 94: 245-248

2 Baldo MA, O'Brien DF, You Y, Shoustikov A, Sibley S, Thompson ME, Forrest SR. Highly efficient phosphorescent emission from organic electroluminescent devices. Nature, 1998, 395: 151-154

3 Kawamuray Y, Goushi K, Brooks J, Brown JJ, Sasabe H, Adachi C. 100\% phosphorescence quantum efficiency of Ir(III) complexes in organic semiconductor films. Appl Phys Lett, 2005, 86: 071104-071107

4 Lamansky S, Djurovich P, Murphy D, Abdel-Razzag F, Lee HE, Adachi C, Burrows PE, Forrest SR, Thompson ME. Highly phosphorescent bis-cyclometalated iridium complexes: Synthesis, photophysical characterization, and use in organic light emitting diodes. J Am Chem Soc, 2001, 123: 4304-4312

5 Baldom A, O'Brien DF, Thompson ME, Forrest SR. Excitonic singlet-triplet ratio in a semiconducting organic thin film. Phys Rev B, 1999, 60: $14422-14428$

6 Hu DH, Cheng G, Lu P, Liu H, Shen FZ, Li FH, Lv Y, Dong WY, Ma YG. Peripheral cyanohexyl substituent in wide bandgap polymer: Increase the electron injection property for blue phosphorescence light emitting device. Macromolecular Rapid Communications, 2011, 32: $1467-1471$

7 Hu DH, Shen FZ, Liu H, Lu P, Lv Y, Liu DD, Ma YG. Separation of electrical and optical energy gaps for constructing bipolar organic 
wide bandgap materials. Chem Commun, 2012, 48: 3015-3017

8 Hu DH, Lu P, Wang CL, Liu H, Wang H, Wang ZM, Fei T, Gu X, Ma YG. Silane coupling di-carbazoles with high triplet energy as host materials for highly efficient blue phosphorescent devices. J Mater Chem, 2009, 19: 6143-6148

9 Liu H, Cheng G, Hu DH, Shen FZ, Lv Y, Sun GN, Yang B, Lu P, Ma YG. A highly efficient, blue-phosphorescent device based on a wide-bandgap host/FIrpic: Rational design of the carbazole and phosphine oxide moieties on tetraphenylsilane. Adv Funct Mater, 2012, 22: $2830-2836$

10 O'Brien DF, Baldo MA, Thompson ME, Forrest SR. Improved energy transfer in electrophosphorescent devices. Appl Phys Lett, 1999, 74: 442-444

11 Adachi C, Baldo MA, Thompson ME, Forrest SR. Nearly 100\% internal phosphorescence efficiency in an organic light-emitting device. $J$ Appl Phys, 2001, 90: 5048-5051

12 Baldo MA, Adachi C, Forrest SR. Transient analysis of organic electrophosphorescence II transient analysis of triplet-triplet annihilation. Phys Rev B, 2000, 62: 10967-10977

13 Kwong RC, Lamansky S, Thompson ME. Organic light-emitting devices based on phosphorescent hosts and dyes. Adv Mater, 2000, 12: $1134-1138$

14 Lamansky S, Kwong RC, Nugent M, Nugent M, Djurovich PI, Thompson ME. Molecularly doped polymer light emitting diodes utilizing phosphorescent Pt(II) and Ir(III) dopants. Org Electron, 2001, 2: 53-62

15 Noh YY, LEE CL, KIM JJ, Yase K. Energy transfer and device performance in phosphorescent dye doped polymer light emitting diodes. $J$ Chem Phys, 2003, 118: 2853-2864

16 Kim TH, YOO DH, PARK JH, Park OO, Yu JW, Kim JK. Enhanced electrophosphorescence via highly efficient energy transfer from conjugated polymer. Appl Phys Lett, 2005, 86: 171108-171110

17 Fei T, Cheng G, Hu DH, Lu P, Ma YG. A wide band gapo polymer derived from 3,6-carbazole and tetraphenylsilane as host for green and blue phosphorescent complexes. J Polym Sci Part A: Polym Chem, 2009, 47: 4784-4792

\title{
The synthesis of phosphonate-functionalized tetraphenylsilane unit and its application in wide band-gap polymer materials
}

\author{
WANG Chu, LIU He, HU DeHua, LU Ping*', MA YuGuang \\ State Key Laboratory of Supramolecular Structure and Materials, Jilin University, Changchun 130012, China \\ *Corresponding author (email: lup@jlu.edu.cn)
}

\begin{abstract}
Phosphonate-functionalized tetraphenylsilane group was designed and synthesized. Coupling with 3,6-substituted carbazole, wide band-gap polymer host SiCzP was synthesized through Suziki reation by using above designed group as fundamental unit. The structure were systematically characterized. Compared with model polymer $\mathrm{SiCz}$, the strong electron-withdrawing property of phosphonate unit could decrease the LUMO energy level and facilitate electron injection. The glass transition temperature and decompostion temperature of $\mathrm{SiCz} \mathrm{P}$ and $\mathrm{SiCz}$ were 219 and 227, 441 and $426{ }^{\circ} \mathrm{C}$, respectively. Both compounds had good film stability. The brightness and efficiency of doped devices were both increased by the introduction of phosphonate unit and the maximun current efficiency and power efficiency were imporved by $98 \%$ and $75 \%$, respectively.
\end{abstract}

Keywords: wide band-gap, polymer, host materials, Suzuki reaction 